\title{
Análisis histórico de la Reforma Psiquiátrica andaluza: los inicios y el proyecto
}

\author{
José Carmona Calvo \\ Francisco del Río Noriega \\ Servicio Andaluz de Salud
}

\begin{abstract}
Resumen
Se expone en las páginas siguientes el proceso de transformación de los recursos psiquiátricos habido en la región de Andalucía, España. En este artículo se describe en primer lugar, las transformaciones realizadas en los últimos años de la etapa franquista, y cuya iniciativa se debió casi exclusivamente a las actividades organizadas por los profesionales frente a las Administraciones de las que dependían. En segundo lugar, el período posterior a la instauración de la democracia y que permitió y favoreció el desarrollo de políticas sanitarias que diseñaron e implantaron nuevos modelos de atención sanitaria general y de salud mental en particular.
\end{abstract}

Palabras claves: reforma psiquiátrica; salud mental comunitaria; hospital psiquiátrico; modelo de servicios en salud mental.

\begin{abstract}
Historical analysis of the psychiatric reform in Andalucia: the beginning and the proposed. The following paper talks about the transformation process of psychiatrics resources in Andalousia, Spain. This article firstly describes the transformations happened in the last years of the Franco disctatorship and whose initiatives were set, almost exclusively, by the professionals, instead of the authorities which they were depending on. Secondly, the following period to the establishment of the democracy allowed and helped the development of sanitary politics that draw and introduced new methods of sanitary general attention and particularly of mental health.
\end{abstract}

Keywords: psychiatric reform; communal mental health; psychiatric hospital; model of mental health services.

$\mathrm{L}$ os procesos de reformas de la salud mental comenzaron en España en la década de los años 1980. Pero las desigualdades en su inicio, su recorrido y sus resultados en las distintas regiones (Comunidades Autónomas) que componen el Estado Español, han condicionado que no exista un único modelo asistencial. Todas ellas han mantenido como línea maestra la integración del paciente en la sociedad mediante una metodología de trabajo comunitario. Sin embargo, sólo en algunas Comunidades Autónomas, como en Andalucía, Asturias y Navarra han desaparecido los Hospitales psiquiátricos y sustituidos por un conjunto de servicios sanitarios y sociales comunitarios. En otras, se ha apostado por la mejora de las condiciones estructurales, asistenciales y de respeto a los derechos humanos en los Hospitales psiquiátricos, coexistiendo con dispositivos comunitarios de nueva creación.

Estos artículos, en su parte I y II, son el resultado de una revisión del capítulo sobre la Reforma Psiquiátrica Andaluza, en el que participamos como autores, e incluidos en la ponencia "Dos décadas tras la Reforma Psiquiátrica" del XXIV Congreso de la Asociación Española de Neuropsiquiatría en 2006 (Pérez, 2006). Se trata pues de un estudio descriptivo del camino complejo que ha seguido la transformación de los servicios de atención a la salud mental en Andalucía, y que por su dimensión los hacemos en dos artículos. Consideramos como eje de la reforma en Andalucía la institución creada a tal fin: el Instituto Andaluz de Salud Mental (IASAM). Dedicamos el primer artículo a los inicios y el proyecto reformista: con una etapa preIASAM de reformas parciales y aisladas, hasta 1984, y el período de funcionamiento del Instituto, de 1984 a 1991. Y el segundo artículo al período pos-IASAM, de 1991 a 2001, y una última época 2001 a 2011, a la que denominaremos etapa de la Salud Mental Reformada. Finalmente, recordar que Andalucía es una región que ocupa el sur de España. Tiene una extensión de unos $87.000 \mathrm{~km}^{2}$ y 8.350 .000 habitantes en la actualidad $(6.800 .000$ al inicio de la reforma) y está divida en ocho provincias.

\section{Etapa pre-IASAM}

Tiene lugar en los últimos años del período franquista y en un contexto general de modernización y democratización de todas las estructuras del Estado Español. Los recursos de atención a los trastornos mentales existentes, especialmente los ligados a los hospitales psiquiátricos, estaban cuestionados en su 
cantidad y en la calidad de su funcionamiento y sus objetivos por gran parte de la comunidad científica, quienes demandaban su sustitución o transformación por un modelo asistencial centrado en la comunidad.

\section{Experiencias de reformas en las provincias de Anda- lucía}

Las experiencias de reformas, o pre reformas, tienen características propias en cada una de las provincias. Podemos señalar las siguientes en un período anterior a 1980-1981:

HUELVA (González Álvarez \& Fernández Sanabria, 1980). La experiencia de transformación se inicia a partir de la puesta en funcionamiento en 1971 de un hospital psiquiátrico provincial que se provee con pacientes que proceden de otros hospitales psiquiátricos (Miraflores - en Sevilla - y Ciempozuelos - en Madrid -). Los profesionales adscritos, tras un análisis de esta situación, hacen las siguientes propuestas asistenciales:

- Esta iniciativa debía tener como objetivo final el dotar a la provincia de una asistencia psiquiátrica según conocimientos actualizados (atención continuada, comprensiva y comunitaria). Así, el resultado final debía corresponderse al modelo de la Psiquiatría de Sector.

- Reorganización interna del centro, en la línea teórica de la Comunidad Terapéutica.

- El presupuesto básico es que existen condiciones objetivas materiales-humanas, y subjetivas (motivación del personal) para realizarlo.

- Se solicita aumentar las horas de dedicación del personal del centro (eran sólo 4 horas el personal técnico titulado) y el aumento salarial a todas las categorías del personal.

- Distribución de los pacientes con arreglo a criterios clínicoasistenciales, superando los criterios económicos y sexuales.

- Organización del personal en equipos multidisciplinarios.

- Introducción de reuniones de equipo, reuniones comunitarias de personal y pacientes, potenciación de las terapias colectivas, reorganización de las consultas externas, etc.

Si bien estas líneas son aceptadas y apoyadas en un principio, desarrollándose a lo largo de 1973-1974, pronto se ven recortadas y obstaculizadas desde la Administración provincial. No obstante, el movimiento de profesionales, a pesar de las dificultades, logra hacia 1980 que prácticamente todas las propuestas señaladas progresivamente se fueran imponiendo. Así, en esta fecha los datos asistenciales se ven modificados. De un Hospital psiquiátrico de 350 camas y con el único objetivo de ser una institución cerrada, se obtienen los siguientes resultados:

- Se utilizaron en estos años sólo 270 camas, del total disponible, y al final del período habían descendido a 212. Los pacientes de larga estancia, desde el principio, fueron ubicados en lugares diferentes según patología y objetivos diferentes: la rehabilitación para pacientes con trastornos mentales graves y el asilamiento para pacientes oligofrénicos y seniles.

- La actividad interna con los pacientes es ampliada y diversificada: intervenciones psicoterapéuticas diversas, ocupacionales, ocio, participación de los pacientes a través de reuniones conjuntas de personal y pacientes, etc.

- Los profesionales se desplazan a diversos centros y localidades de la provincia para realizar una atención extra hospitalaria, que pasa desde las 566 intervenciones en 1972, a las 5.722 de 1979.

MALAGA (Corcés Pando \& del Río, 1980). El Hospital psiquiátrico de Málaga era un centro construido en el siglo XIX y por tanto con todas las características propias de ésta época, especialmente en la infraestructura pero también en sus hábitos. Pues bien, coincidiendo con el intento de trasladarlo a otra población distinta y que suponía mayor marginación, se inicia un movimiento de protesta de los profesionales impidiéndolo. Se aprovecha para modificar la actividad asistencial, en especial las dirigidas a la rehabilitación de la población hospitalizada largos años, la mayoría de la población ingresada (531 pacientes en 1979). Los cambios asistenciales se dirigen a mejorar los autocuidados y la autonomía de los pacientes y a facilitar la externalización y reinserción de muchos de ellos en recursos externos de diverso origen. A través del trabajo comunitario de los profesionales del propio hospital psiquiátrico en colaboración con otros recursos asistenciales, sanitarios y no sanitarios, y otros de carácter voluntario, fue posible realizar este proceso. El fruto de un año de trabajo en esta dirección se tradujo en que 120 pacientes encontraron alojamiento alternativo al Hospital psiquiátrico. Además, se pusieron en marcha varias consultas externas en Málaga, en Vélez y en Antequera, atendiendo a más de 2.400 consultas.

SEVILLA (Piñero Ramirez, Mendez Marín, \& Murillo Esteve, 1980). En el período que se contempla, las mejoras que se llevan a cabo en este Centro parten de las reclamaciones de los Auxiliares Psiquiátricos, en principio exclusivamente laborales, e iniciadas en torno a 1976. Progresivamente se transforman en la exigencia de un perfil profesional para este colectivo, reivindicando una formación adecuada para el cuidado de los pacientes que atendían. Estas peticiones incluían la exigencia de una mejora de la atención sanitaria y de las condiciones de vida de éstos. La movilización de este colectivo se transmitió progresivamente. En primer lugar, a los pacientes quienes se unen a las reivindicaciones económicas para la remuneración de actividades supuestamente ocupacionales en el hospital psiquiátrico, pero que eran realmente actividades laborales sin retribuir y con una clara rentabilidad para el centro. Se produce así, un movimiento de apoyo mutuo entre ambos colectivos. En segundo lugar, más tardíamente, se movilizaron los profesionales técnicos. Como emergente de esta situación, radio Sevilla proclamó Sevillanos del año 1978, en el apartado social, al trabajo del equipo denominado "Trabajadores de la Salud Mental", por la actividad denominada "Salta la Tapia". Esta iniciativa consistía en actividades organizadas en el Hospital psiquiátrico de Miraflores y compartidas entre pacientes, profesionales y personas externas, con el objetivo de favorecer el conocimiento mutuo y la reintegración social de los pacientes.

Con ser estas tres experiencias las más importantes, en el período posterior a 1980-1981 y hasta el momento de la creación del IASAM en todas las provincias existieron experiencias de transformación de los hospitales psiquiátricos. En todas ellas y favorecido por el cambio político se consolidaron los proyectos de reforma de los hospitales psiquiátricos en las otras provincias: 
Jaén, Cádiz, Córdoba, Granada y Almería. Siempre, basándose en los presupuestos de la Psiquiatría Comunitaria, como en la mayoría de los movimientos de transformación de otros países del mundo. Sin embargo, no va a ocurrir así con el resto de los otros recursos ligados a otras instituciones de atención a problemas de salud mental, como los dependientes de la AISNA o el INSALUD, instituciones de ámbito estatal, que permanecieron sin cambios hasta que el IASAM se hizo cargo del proceso de transformación de todos los recursos existentes.

\section{El marco socio-político de los años 1980-84: la au- tonomía andaluza}

Esta etapa estuvo marcada por las condiciones de carácter político y administrativas existentes en el resto del estado. La diferencia la experiencia andaluza con otras comienza por las primeras elecciones democráticas municipales del 1979 en las que, merced a un pacto de los partidos de izquierda (Partido Socialista Obrero Español, Partido Comunista Español y Partido Socialista Andaluz), se hizo posible un gobierno homogéneo en los ocho gobiernos provinciales (Diputaciones), y que propusieron como uno de sus objetivos transformar los servicios sanitarios de beneficencia adscritos a estas administraciones en servicios sanitarios públicos y de cobertura universal. A esta circunstancia se añade la adquisición de autogobierno para la autonomía andaluza y, con ella, la capacidad de gestionar los servicios sanitarios públicos. En este ámbito, las primeras elecciones autonómicas dieron la victoria al Partido Socialista Obrero Español, por lo que todas las administraciones con competencias en salud tuvieron un mismo color político. Esta coyuntura permitió encarar con optimismo una etapa de transformación de los servicios de salud mental en Andalucía.

\section{La reforma sanitaria y las recomendaciones de la Comisión Nacional para la Reforma Psiquiátrica}

El autogobierno permitió intervenir sobre la dispersión de los recursos sanitarios, la diversidad de redes, los diferentes modelos asistenciales, los déficits de recursos, etc. Derivado de esta realidad se impuso como necesario llevar a cabo un proceso de reforma sanitaria general. Este proceso se inicia en 1982, con la formulación de una serie de objetivos a cubrir (Instituto Andaluz de Salud Mental, 1988a):

- La integración de las distintas redes administrativas y de servicios.

- La creación de una única estructura de gestión: el Servicio Andaluz de Salud.

- La modificación del modelo tradicional de servicios sanitarios, a través de:

- Una nueva territorialización;

- La remodelación y potenciación de la Atención Primaria de Salud;

- La reforma del sistema de asistencia hospitalaria y especializada.

- El diseño y desarrollo de Programas de Atención.

- El incremento y formación de los recursos humanos.

- La racionalidad del gasto sanitario adecuándolo a las nuevas prioridades.

En éste contexto general se inicia en Andalucía el proyecto de transformación de los recursos existentes para la atención a los trastornos mentales. A este proyecto se incorporan las recomendaciones de la Comisión Nacional para la Reforma Psiquiátrica, de nivel estatal.

Etapa IASAM: 1984-1991

\section{Contexto y objetivos en la creación del Instituto An- daluz de Salud Mental.}

Sin pretender realizar un análisis exhaustivo, el diagnóstico de situación de la atención en salud mental que recibían entonces los andaluces no puede ser otro que la inadecuación de los recursos e intervenciones a las necesidades reales de la población. Los datos cualitativos y cuantitativos disponibles eran insuficientes y poco fiables, tanto los referidos a la salud mental como al conjunto de la atención sanitaria. De forma sucinta puede esquematizarse la situación de partida teniendo en cuenta la existencia de una gran multiplicidad y descoordinación de redes públicas. Había cinco administraciones con competencias en salud mental: Diputaciones Provinciales, Red de Asistencia Sanitaria de la Seguridad Social de Andalucía (RASSSA), Administración Institucional de la Sanidad Nacional (AISNA), Hospitales Clínicos Universitarios y Instituto Social de la Marina.

Esta fragmentación de competencias favorecía la separación del resto del Sistema Sanitario General que percibía a la salud mental como algo ajeno a lo sanitario y cuya integración en el conjunto no era del todo comprendida y significaba un problema de difícil solución. Por otro lado, la valoración profesional y social de sus recursos era negativa. De manera que, los profesionales, como los pacientes, eran también estigmatizados. Por otra parte, la distribución territorial de los escasos recursos, públicos y privados, era muy desigual, la mayoría se concentraba sobre todo en las capitales de provincias, dejando las zonas rurales desatendidas y con grandes dificultades de transporte para el desplazamiento.

En este contexto, a cuyo impulso no fue ajeno el que el Viceministro de Salud fuera un psiquiatra, se comenzó en Andalucía en 1984 un proceso de transformación de las estructuras públicas de atención a la salud mental como parte diferenciada de un amplio movimiento de reforma sanitaria. Como culminación de ésta se llega a la constitución de una única estructura sanitaria pública (el Servicio Andaluz de Salud), poniendo el énfasis en el desarrollo de servicios de atención primaria (López, Benítez, García, \& Weissman, 1998). En este marco de reforma general de la atención sanitaria andaluza, tiene lugar el proceso de Reforma Psiquiátrica, que comienza con la puesta en marcha del IASAM. Este paso tuvo en el momento de su inicio un fuerte apoyo político de todas las Instituciones de las que dependían recursos de atención a la salud mental existentes y una adhesión considerable de la mayor parte de los trabajadores de atención a la salud mental.

Los objetivos Generales del IASAM fueron:

- Unificar el conjunto de recursos sanitarios de la atención a la salud mental.

- Introducir elementos progresivamente más complejos y ajustados en la planificación y programación de las actuaciones, 
a medida que se pudiera hacer un diagnóstico más exacto de las necesidades de atención, ya que fue imposible realizarlos en los inicios, por la lamentable falta de datos.

- Modificar la estructura de servicios sustituyendo al Hospital psiquiátrico por una red diversificada, de cobertura general a toda la población, articulada según niveles de complejidad y especialización, de base y orientación comunitaria, y integrada en el Servicio Andaluz de Salud.

- Incrementar el número, diversidad y cualificación de los profesionales.

\section{Aspectos cuantitativos de la reforma: la situación de partida:}

Los servicios. En este aspecto solo puede resaltarse la escasez e inadecuación de los recursos públicos/privados para realizar la tarea. La dotación total se resume en la tabla 1: 8 Hospitales psiquiátricos tradicionales, con infraestructura muy deteriorada, aislados muchos de ellos de núcleos de población y con recursos humanos de escasa cualificación, con una capacidad cercana a las 4000 camas de las que se mantenían ocupadas unas 3000 , correspondiendo 2700 a pacientes con tiempos de estancia superiores a 6 meses y las 300 camas restantes a pacientes con estancias más cortas; 69 consultas de neuropsiquiatría, en las que se atendía la patología neurológica y psiquiátrica por un solo facultativo durante 2,5 horas/día; 8 Centros de Higiene Mental de la AISNA, con recursos y tiempo de dedicación, igualmente, muy escasos; y sólo existían dos Hospitales Universitarios con dotación de camas para pacientes psiquiátricos agudos.

Otros recursos existentes derivados de los procesos de transformación iniciados antes de esta etapa o con los que

Tabla 1

Servicios psiquiátricos en Andalucía en 1984(6)

\begin{tabular}{lcccc}
\hline $\begin{array}{c}\text { Hospitales } \\
\text { Psiquiátricos } \\
\text { Públicos }\end{array}$ & Camas & $\begin{array}{c}\text { Hospitales } \\
\text { Psiquiátricos } \\
\text { Privados }\end{array}$ & $\begin{array}{c}\text { Consultas de } \\
\text { Neuropsiquiatría }\end{array}$ & Centros de AISNA \\
\hline Almería & 400 & No & 4 & 1 \\
Cádiz & 650 & No & 8 & 2 \\
Córdoba & 550 & No & 8 & 1 \\
Granada & 320 & No & 10 & 2 \\
Huelva & 250 & No & 4 & 1 \\
Jaén & 500 & No & 7 & 2 \\
Málaga & 420 & 3 & 11 & 2 \\
Sevilla & 1.200 & 1 & 17 & 12 \\
Andalucía & 4.290 & 4 & 69 & 2 \\
\hline
\end{tabular}

Nota. Fuente: Instituto Andaluz de Salud Mental

contaban otras instituciones como la Universidad se concretaban en 20 Equipos de Salud Mental, 2 Unidades de Salud Mental Infantil, 1 Hospital de Día y 4 Unidades de Psiquiatría situadas en Hospitales Generales con 144 camas de corta estancia. Evidentemente la institución más importante y con más hegemonía, la constituían sin duda los hospitales psiquiátricos, "cuya oferta asistencial" incluía, casi exclusivamente, la atención asilar de pacientes con trastornos mentales graves e importantes carencias sociales.

\section{Los pacientes.}

La población hospitalizad. Como hemos señalado anteriormente, las características de la demanda de salud mental de la población en ese momento solo pueden inferirse. Prácticamente solo se tienen datos fiables de la población asistida en la institución manicomial. Se trataba de una población de edad media avanzada, de predominio masculino, en su mayoría compuesta por solteros y de un perfil educativo y socioeconómico muy bajo. Diagnosticados en su mayor parte de psicosis y deficiencia mental, pero con una clínica predominantemente "residual" y "asintomática", con escasa presencia de sintomatología activa (sólo se recoge en el $17 \%$ de los pacientes) y con una importante patología somática asociada. Todo ello refuerza la tesis de que los hospitales psiquiátricos cubrían sobre todo las necesidades de atención social, realizando la función de almacén de la marginalidad. La tabla 2 recoge las características de la población asilada al inicio de la trayectoria del IASAM:

La distribución diagnostica de los pacientes ingresados redunda, así mismo, en el uso asilar de los hospitales psiquiátricos. Como muestra la tabla 3 , los pacientes de larga estancia son, casi en un $80 \%$, trastornos mentales graves, cronificados y sin expectativas de futuro y deficientes mentales. Los pacientes de corta estancia tienen una distribución diagnostica algo diferente. Los pacientes psicóticos siguen siendo mayoría porcentual frente a las toxicomanías, trastornos afectivos y trastornos neuróticos que conforman aproximadamente el $40 \%$ de pacientes hospitalizados con estancias inferiores a 6 meses.

Respecto a esta población asilada no hay que olvidar las condiciones de vida que soportaba en relación a sus derechos fundamentales. Para evaluarla se creó a tal efecto una comisión (Instituto Andaluz de Salud Mental, 1985), y sus conclusiones fueron las siguientes: insuficiente garantía legal de los derechos fundamentales de los internos; existencia de anomalías jurídicas y asistenciales con pacientes no necesitados de tratamiento psiquiátrico, o, en cualquier caso, de internamiento en tales centros; situación de práctica indefensión de la población internada en cuanto a estas limitaciones de sus derechos; ausencia de regulación legal de la actividad laboral de los internados; desempeño, en la práctica, de funciones asilares y de cobertura social y no terapéutica por parte de los hospitales psiquiátricos; y actuación de la estructura asistencial como factor de cronificación 
Tabla 2

Características de la población de larga estancias en hospitales psiquiátricos (1985)

\begin{tabular}{|c|c|c|}
\hline \multicolumn{3}{|c|}{ Numero de pacientes } \\
\hline Corta estancia & & 315 \\
\hline Larga estancia & & 2672 \\
\hline Totales & & 2987 \\
\hline \multicolumn{3}{|c|}{ Características de los residentes de larga estancia } \\
\hline Edad & \multicolumn{2}{|c|}{ Media de 54 anõs; $>65$ años, $30 \%$} \\
\hline Nivel educativo & \multicolumn{2}{|c|}{$76 \%$ analfabetos $y / 0$ sin estudos } \\
\hline \multirow[t]{2}{*}{ Dignósticos más frecuentes } & Psicosis & $51 \%$ \\
\hline & Deficientes mentales & $30 \%$ \\
\hline Tiempo de estancia & \multicolumn{2}{|c|}{ Média: 13 años; $25 \%>20$ años } \\
\hline \multirow[t]{3}{*}{ Sintomatologia psiquiátrica } & Activa & $17 \%$ \\
\hline & Residual & $59 \%$ \\
\hline & Asintomática & $23 \%$ \\
\hline Situación somática & \multicolumn{2}{|c|}{$\begin{array}{l}51 \% \text { con algún tipo de alteración } \\
12 \% \text { con alteraciones graves }\end{array}$} \\
\hline
\end{tabular}

Tabla 3

Población hospitalizada en instituciones psiquiátricas en Andalucía (enero 1985): distribución diagnostica

\begin{tabular}{lcccc}
\hline & \multicolumn{2}{c}{ Población de corta estancia $^{\mathrm{a}}$} & \multicolumn{2}{c}{ Población de larga estancia $^{\mathrm{b}}$} \\
\cline { 2 - 5 } Grupos diagnosticos & $\mathrm{n}$ & \multicolumn{1}{c}{$\%$} & $\mathrm{n}$ & $\%$ \\
\hline Psicosis Orgánicas & 31 & 6,5 & 176 & 6,3 \\
Psicosis Delirantes & 204 & 42,6 & 1.327 & 47,2 \\
Cuadros Afectivos & 72 & 15,0 & 82 & 2,9 \\
Transtornos Neuróticos & 60 & 12,5 & 68 & 2,4 \\
Alcoholismo y Drogodependencias & 62 & 12,9 & 121 & 4,3 \\
Epilepsia & 2 & 0,4 & 132 & 4,7 \\
Retraso mental & 29 & 6,0 & 832 & 29,6 \\
Otros & 19 & 3,9 & 74 & 2,6 \\
Totales & 479 & 100,0 & 2.812 & 100,0 \\
\hline
\end{tabular}

Nota. Fuente: Instituto Andaluz de Salud Mental

${ }^{a}$ Estancia inferior a 6 meses (desde el último ingreso)

${ }^{\mathrm{b}}$ Estancia superior a 6 meses (desde el último ingreso)

en la mayoría de los casos.

La demanda en la población no hospitalizada. Las dificultades teóricas y metodológicas para realizar una aproximación real a los problemas de salud mental de los andaluces eran aun mayores cuando se trataba de obtener datos asistenciales procedentes del ámbito no hospitalario. Realizar una planificación asistencial resultaba azaroso, pues para establecer la prevalencia e incidencia de muchas patologías fue necesario recurrir a extrapolaciones de estudios realizados en otras comunidades, muy alejadas cultural y geográficamente y/o en datos de la población general. La tabla 4 recoge un muestrario de la realidad asistencial en ese momento.

Los datos de frecuentación de algunos servicios de neuropsiquiatría ofrecían una elevada prevalencia de "trastornos neuróticos". A estos cuadros le seguían a distancia los trastornos afectivos y los psicóticos, para los cuales no existían prácticamente de recursos adecuados para su tratamiento. Las mismas dificultades se planteaban para establecer el volumen de actividad asistencial, cuya única prestación era ésta consulta de neuropsiquiatría con sólo tratamiento farmacológico (tabla 5).

Los profesionales. La dotación de recursos humanos que atendían la totalidad de éstos dispositivos en 1985 era manifiestamente deficitaria y resultaba inoperante para realizar una atención interdisciplinar básica, para cerca 6.789.772 habitantes (Instituto de Estadística y Cartografia de Andalucía, 1986) (tabla 6):

Resulta evidente la escasa presencia de facultativos, especialmente de psicólogos cuyo número es casi anecdótico en las instituciones sanitarias públicas de ese momento. Por otra parte, conviene recordar que el número de psiquiatras, cuya tasa en ese momento representa un 3'86 por 100.0000 habitantes, debe ser relativizado, por varias razones: por su ubicación, casi 
Tabla 4

Consultas ambulatorias de neuropsiquiatría en Andalucía: distribucion diagnostica de la demanda atendida $(1986)^{a}$

\begin{tabular}{lcc}
\hline \multicolumn{1}{c}{$\begin{array}{c}\text { Diagnosticos } \\
\text { (grandes grupos) }\end{array}$} & $\begin{array}{c}\text { Pacientes } \\
\mathrm{n}\end{array}$ & $\begin{array}{c}\text { Atendidos } \\
\%\end{array}$ \\
\hline Psicosis Orgánicas & 22 & 1,6 \\
Psicosis Delirantes & 46 & 3,3 \\
Cuadros Afectivos & 257 & 18,6 \\
Trastornos Neuróticos & 328 & 23,8 \\
Alcoholismo y Drogodependencias & 39 & 2,8 \\
Problemas Psicosomáticos & 51 & 3,7 \\
Trastornos infantiles & 32 & 2,3 \\
Retraso mental & 27 & 1,9 \\
Problemas neurológicos & 469 & 34,0 \\
Otros (mal definidos o sin diagnostico) & 109 & 7,9 \\
Totales & 1.380 & 100,0 \\
\hline Nota. Muestra de 1.380 consultas realizadas en la 3 ${ }^{\text {a semana de Mayo }}$ \\
Fuente: Instituto Andaluz de Salud Mental (1986). Estudio de la demanda de las consultas de \\
Neuropsiquiatría en Andalucia. Sevilla: Consejería de Salud y Servicios Sociales
\end{tabular}

Tabla 5

Consultas ambulatorias de neuropsiquiatría en Andalucía: actividad asistencial en 1986

\begin{tabular}{lcccccc}
\hline Provincias & Neuropsiquiatras & $\begin{array}{c}\text { Consultas/día } \\
\text { por } \\
\text { neurpsiquiatra }\end{array}$ & $\begin{array}{c}\text { Consultas } \\
\text { anuales }\end{array}$ & $\begin{array}{c}\text { Consultas } \\
\text { anuales } \\
\text { tasa x 1000 } \\
\text { hab. }\end{array}$ & $\begin{array}{c}\text { Primeras } \\
\text { consultas } \\
\text { anuales }\end{array}$ & $\begin{array}{c}\text { Primeras } \\
\text { consultas } \\
\text { tasa x 1000 } \\
\text { hab. }\end{array}$ \\
\hline Almería & 4 & 23.53 & 25.196 & 61,02 & 14.845 & 34,14 \\
Cádiz & 8 & 25.46 & 50.509 & 52,16 & 17.890 & 18,20 \\
Córdoba & 8 & 13.69 & 26.452 & 37,77 & 11.999 & 17,19 \\
Granada & 10 & 12.45 & 31.091 & 41,20 & 12.045 & 15,90 \\
Huelva & 4 & 14.43 & 12.869 & 32,67 & 4.864 & 12,26 \\
Jaén & 7 & 11.89 & 20.549 & 34,66 & 8.209 & 13,14 \\
Málaga & 11 & 16.70 & 44.348 & 43,06 & 22.068 & 21,36 \\
Sevilla & 17 & 17.81 & 80.312 & 52,52 & 23.884 & 17,82 \\
Andalucía & 69 & 16.93 & 291.326 & 44,82 & 115.804 & 17,82 \\
\hline
\end{tabular}

Nota. Fuente: Consejería de Salud, 1986

Tabla 6

Personal adscrito a los servicios psiquiátricos públicos en Andalucía (1984)

\begin{tabular}{|c|c|c|c|c|c|}
\hline $\begin{array}{c}\text { Categorías } \\
\text { profesionales }\end{array}$ & Diputaciones & Universidad & $\mathrm{AISN}^{\mathrm{a}}$ & RASSSA & Total \\
\hline Psiquiatras $^{\mathrm{b}}$ & 112 & 30 & 21 & 83 & 246 \\
\hline Psicólogos & 19 & 1 & 12 & - & 32 \\
\hline $\begin{array}{l}\text { Terapeutas } \\
\text { sociales }\end{array}$ & 33 & 1 & 7 & - & 41 \\
\hline $\begin{array}{l}\text { Enfermeros } \\
\text { diplomados }\end{array}$ & 95 & 15 & 7 & - & 1.218 \\
\hline Auxiliares & 1.198 & 20 & - & - & 1.218 \\
\hline Otros $^{c}$ & 589 & 3 & 9 & - & 601 \\
\hline Totales & 2.046 & 70 & 56 & 83 & 2.255 \\
\hline
\end{tabular}

siempre en los Hospitales psiquiátricos, por las condiciones laborales de ejercicio profesional (jornadas de escasas horas de dedicación) y por el pluriempleo imperante; de manera que, usualmente, un psiquiatra ocupaba varias plazas asistenciales en la misma provincia. La variedad de dependencias administrativa de los distintos servicios complicaba extraordinariamente, incluso impedía, el intercambio de información y una coordinación mínima entre ellos. Lo mismo puede aplicarse al número de enfermeros diplomados y trabajadores sociales.
Destaca por su número, claramente desproporcionado con los otros colectivos, los auxiliares de enfermería o cuidadores. Eran el grueso de los trabajadores de los hospitales psiquiátricos, en condiciones de contratación, formación y capacitación muy dispares. Es difícil evaluar, en todos los colectivos implicados en la asistencia psiquiátrica de ese momento, la cualificación real que poseían, aunque era evidente que estaban necesitados de un importante reciclaje para adecuarse a la incipiente reforma, por otra parte ineludible para poder realizar el proceso 
de transformación que las instituciones a las que pertenecían necesitaban.

\section{Aspectos cualitativos: el modelo y el desarrollo del modelo.}

El protagonismo de los profesionales. La llegada del IASAM pone en marcha una nueva política sanitaria. Los postulados de la Reforma Psiquiátrica iniciada implican un autentico cambio epistemológico en el modo de atender y conceptuar los problemas de salud mental que va más allá de una simple reordenación e incremento de los recursos en el marco de la asistencia psiquiátrica.

El nuevo modelo se asienta resumidamente sobre un concepto de salud integral que trasciende la idea de salud como ausencia de enfermedad, que se compone de los elementos psicológico, biológico y social, y va desde la prevención de los trastornos hasta la rehabilitación e integración social de los pacientes. Desde la nueva perspectiva, resulta imposible hacer una atención del problema dando respuestas exclusivamente "biológicas" y "especializadas" y fuera del medio natural del paciente. Ésta era la única respuesta posible desde el hospital psiquiátrico. El documento de trabajo $\mathrm{n}^{\mathrm{o}} 1$ editado por el IASM en julio de 1988: "Bases generales para la atención comunitaria en Salud Mental" (Instituto Andaluz de Salud Mental, 1988b) señala el siguiente cuádruple deslizamiento en el centro de interés en el abordaje de los problemas psíquicos: 1) Del campo de la recuperación de la salud mental, al de la prevención de la enfermedad; 2) De la psicosis como principal objeto asistencial, a todo el espectro de la psicopatología; 3) Del hospital psiquiátrico como espacio privilegiado, de atención al ámbito comunitario; 4) Del abordaje especializado del problema, a su globalización a través de un primer enfoque no especializado y, a veces, ni siquiera sanitario.

En resumen, el proceso tiene como principal objetivo desmantelar el anterior y obsoleto modelo de atención manicomial, hospitalocentrico, reduccionista y de exclusión social para dar paso a un modelo de atención comunitaria descentralizado, integracionista, interdisciplinario y de intervención comunitaria. El instrumento diseñado para producir el cambio fue el IASAM.

El modelo asistencial implantado se organizó en varios niveles de intervención, con características propias y funciones específicas, estrechamente interrelacionados:

- Nivel de intervención no sanitario formado por la comunidad, sus instituciones y sus redes de recursos sociales formales o informales.

- Nivel de intervención sanitaria básica o primer nivel formado por los equipos de atención primaria en esos momentos en plena fase de diseño.

- Nivel de intervención sanitaria especializada en el ámbito del Distrito Sanitario de Atención Primaria, segundo nivel de asistencia sanitaria para la salud mental, y su eje central para la intervención especializada. Esta formado por los ESMD (equipos de salud mental de distrito). Se quería así generar un tejido asistencial profusamente difundido en la geografía andaluza.

- Nivel de intervención para problemas específicos de salud mental. Con diversas Unidades de Salud mental centradas fundamentalmente en la atención de trastornos mentales graves. Su ámbito geográfico el de un área de salud mental, que comprende generalmente a varios distritos sanitarios.

- Se conforma así una red de atención que queda de la siguiente manera (figura 1):

Se parte de la comunidad, de la familia y del individuo, en coordinación con los Equipos Básicos de Atención Primaria

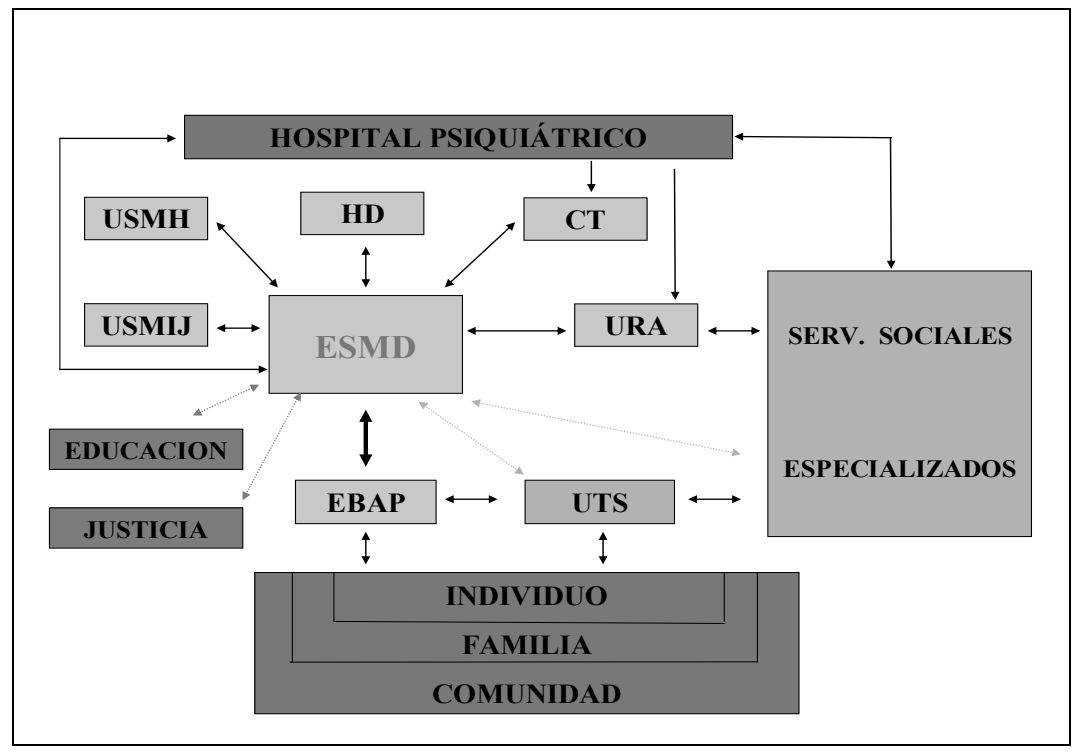

Figura 1. Niveles de intervención en salud mental - Red de cuidado

(EBAP), con las Unidades de Trabajo Social (UTS), con los Servicios Sociales Especializados y con el Servicio de Salud Mental. Los ESMD se consideran centro del modelo y se añaden los servicios de mayor especialización, para áreas específicas. Se describen a continuación todos los dispositivos:

Equipo de salud mental de distrito (ESMD). En otros 
ámbitos Centros de Salud Mental Comunitarios. Son el núcleo del subsistema de salud mental. Responsable de la atención a la población que integra un distrito sanitario, que a su vez comprende varias zonas básicas de salud con sus correspondientes equipos de atención primaria. Su función primordial debe ser ofrecer al conjunto de la población asignada atención sanitaria en salud mental global, ambulatoria, domiciliaria y comunitaria.

En el momento actual, pese a que la distribución geográfica y la diseminación de los ESMD cubren la totalidad del territorio andaluz, con unos cronos aceptables para la mayoría de la población, sus recursos iniciales precisaron un redimensionamiento. En la práctica, la principal dificultad de los ESMD es la desconexión con el primer escalón de la asistencia, puerta de entrada a todo el sistema sanitario. Esto ha permitido en muchos casos la desatención de los profesionales de atención primaria de la casuística de salud mental, colapsando de manera involuntaria la atención en los ESMD y, en definitiva, dificultando el modelo de intervención comunitaria. Existen servicios en los que se han implantado programas funcionales sistematizados, posibilitando un buen ajuste de la demanda, consiguiendo la estabilización de la misma con demoras medias aceptables y favoreciendo la cooperación intersectorial. El Equipo de salud mental, además de asumir las demandas filtradas por los EBAP, dispone de otro nivel de apoyo para la derivación de necesidades más específicas, que comprenden las siguientes unidades asistenciales descritas a continuación.

Unidades de hospitalización en salud mental. Son unidades ubicadas en hospitales generales con una doble función: la atención hospitalaria en régimen de estancias cortas y el desarrollo de programas de enlace con el resto del hospital. Desarrollan programas de atención centrados en situaciones de crisis o agravamiento de la patología que impide al paciente su permanencia en la comunidad. Fueron diseñadas para albergar entre 15 y 30 camas de manera que su estructura permitiera la creación de un ambiente terapéutico. La implantación de estos dispositivos, en número de camas y recursos humanos, puede ser considerada suficiente.

Unidades de salud mental infantil. Concebidas como unidades de apoyo a la atención infantil de los equipos salud mental de distrito, en especial para trastornos graves hasta los 18 años. Su desarrollo ha obligado a reformularlas con un mayor protagonismo de la asistencia directa, ampliando sus programas a la hospitalización, parcial y total e incrementando la accesibilidad con la ampliación del horario. Desarrollan también programas de enlace y apoyo a otros dispositivos sanitarios, educativos, y sociales. Su dotación inicial fue de 11 unidades para toda Andalucía.

Unidades de rehabilitación. Simultanean su función de atención clínica a pacientes como Centro de Día con Programas de Rehabilitación Psicosocial (en zonas urbanas), con la de apoyo a los Equipos de Distrito en el desarrollo de idénticos programas en sus respectivos ámbitos. Destinadas a la atención de pacientes con trastornos psicóticos o de personalidad graves que requieren de intervenciones rehabilitadoras y de reinserción social. A su equipo multidisciplinar común al resto de las unidades incorporan terapeutas ocupacionales y monitores ocupacionales.

Dispositivos experimentales. Al inicio de este proceso de reforma, y con carácter experimental ya que se desconocían las necesidades reales, se diseñan otros dispositivos especializados, destinados a diversificar más la oferta asistencial y formativa "sobre cuyo papel, dentro de una red de servicios como la descrita existe aún factores de incertidumbre que no permiten su inmediata generalización":

Comunidades Terapéuticas. Constituyen estructuras de atención intensiva con hospitalización total de media y larga estancia, para pacientes psicóticos, sin las características de marginación e institucionalismo tradicionalmente asociadas a la "larga estancia" en instituciones psiquiátricas.

Hospitales de Día. Dispositivo de hospitalización parcial para procesos mentales graves de larga evolución, con carácter de recurso intermedio entre la Unidad de Salud Mental del Hospital General y los Equipos de Salud Mental de Distrito.

Unidad de docencia y psicoterapia. Esta unidad se crea orientada a la formación del personal en el tratamiento psicoterapéutico. Organizada en régimen de hospital de día desarrolla programas de atención directa e intensiva a pacientes y a la vez que desarrolla programas de formación e investigación en técnicas psicoterapéuticas. Se ha creado una sola unidad de este tipo hasta el momento. Su actividad ha resultado muy útil especialmente para la formación de postgrado de psicólogos internos residentes y médicos internos residentes de psiquiatría.

\section{Comentarios finales sobre esta etapa}

Los objetivos planteados por el IASAM, se cumplieron prácticamente en su totalidad: Se conformó una sola red y se integraron todos los recursos en el servicio andaluz de salud, lo que puso a las estructuras de atención a la salud mental y a sus profesionales en situación de igualdad con el resto del sistema sanitario. Al final de este período Andalucía contenía los siguientes recursos asistenciales de salud mental (tabla 7).

El número de profesionales adscritos crecieron en número y cualificación (tabla 8 ).

La política intersectorial tuvo un fuerte impulso a través de la realización de reuniones anuales de coordinación con la Administración de Justicia. Sus conclusiones facilitaron y homogeneizaron enormemente el trabajo en todo el territorio, en relación a temas como al internamiento involuntario, el control judicial de los ingresos involuntarios, los informes de judiciales, la situación jurídica de algunos pacientes (penados, incapacitados), etc. Esta colaboración quedó plasmada en protocolos conjuntos de actuación. También se realizaron múltiples grupos de trabajo que muchos casos desembocaron en protocolos conjuntos de actuación con las Fuerzas de Seguridad del Estado, Educación, Trabajo, Asuntos Sociales, etc. Para promover la mejora de la planificación, se creó un sistema 
Tabla 7

Recursos asistenciales en 1990

\begin{tabular}{lcccccc}
\hline Provincias & ESMD & USMIJ & USMHG & $\begin{array}{c}\text { USM } \\
\text { Transitorias en } \\
\text { H. Psiquiátricos }\end{array}$ & $\begin{array}{c}\text { Hospitales } \\
\text { de Día }\end{array}$ & $\begin{array}{c}\text { Unidad de } \\
\text { Docencia y } \\
\text { Psicoterapia }\end{array}$ \\
\hline Almería & 5 & 1 & - & 2 & - & - \\
Cádiz & 6 & - & 1 & 2 & - & - \\
Córdoba & 4 & 1 & 1 & 1 & - & - \\
Granada & 7 & 1 & 1 & 2 & - & 1 \\
Huelva & 5 & 1 & - & 1 & - & - \\
Jaén & 7 & 1 & 1 & - & - & - \\
Málaga & 9 & - & 1 & - & - & - \\
Sevilla & 15 & 2 & 4 & - & 1 & - \\
Andalucía & 58 & 7 & 9 & 8 & 1 & 1 \\
\hline
\end{tabular}

Nota. Fuente: Instituto Andaluz de Salud Mental

Tabla 8

Profesionales adscritos a salud mental

\begin{tabular}{lcc}
\hline Categoría & S.A.S. & \\
Profesionales & 1990 & 1.986 \\
\hline Psiquiatras & 315 & 246 \\
Psicólogos & 184 & 32 \\
Trabajadores Sociales & 134 & 41 \\
Enfermeros & 459 & 117 \\
Auxiliares de Enfermería & 796 & 1.218 \\
Terapeutas Ocupacionales & 25 & - \\
Otros & 116 & 601 \\
Totales & 2.029 & 2.255 \\
\hline
\end{tabular}

Nota. Fuente: Instituto Andaluz de Salud Mental

de indicadores, Sistema de Indicadores de Salud Mental de Andalucía (SISMA), que aún con bastantes imprecisiones, ha conseguido dar una imagen de la demanda y la actividad en salud mental mucho mas más afinada de la que se tenía al inicio de la Reforma.

En cuanto a la formación continuada se realizaron en ese periodo un gran número de cursos y talleres para todos los colectivos de profesionales bajo el patrocinio del IASAM. La formación postgrado recibió igualmente un fuerte impulso, se aumentaron las plazas de MIR de Psiquiatría, de 4 en 1986 a 24 en 1990. En 1986 Andalucía, junto con Asturias, fueron las primeras comunidades autónomas que pusieron en marcha un sistema propio de formación postgraduada para psicólogos, varios años antes de que se pusiera en marcha el programa de formación postgrado de psicología clínica nacional. Todas estas transformaciones, se realizaron con un fuerte apoyo y colaboración de los profesionales (definición de los dispositivos, elección de los indicadores asistenciales, transformación del modelo de historia clínica, encuentros con la justicia, etc). Esta participación fue promovida y alentada por el IASAM, que tuvo como estilo de trabajo incitar y favorecer la colaboración de todos los colectivos en el proceso.

El final del IASAM: crónica de una muerte anunciada. Aspectos positivos y negativos
Sí cualitativamente se cumplieron los objetivos del IASAM no fue así cuantitativamente. La cantidad de recursos tanto humanos y de dispositivos, aunque mejoraron, cuando se disolvió el IASAM en diciembre de 1990, eran todavía muy deficientes para cumplir las necesidades de salud mental de los ciudadanos andaluces. La disolución del IASAM tuvo lugar, una vez concluidas las transferencias al Servicio Andaluz de Salud, de los servicios de todas las instituciones que tenían algo que ver en el tratamiento y/o diagnóstico de las personas con trastornos de salud mental. Por fin la red de atención a la salud mental era única e integrada en el Servicio Andaluz de Salud, como todos los recursos sanitarios. El IASAM fue sustituido por una oficina, incluida en los Servicios Centrales del SAS.

La ley de creación del IASAM contemplaba, en la disposición final, el traspaso de las competencias, funciones y recursos propios de esta institución al futuro SAS (Ley 9/1984). De ahí que, en correspondencia con lo anterior, cuando aparece la ley de creación del Servicio Andaluz de Salud (SAS) el 6 de Mayo de 1986, se recoge en la disposición transitoria primera que en el plazo máximo de tres años, a partir de la promulgación de esta última, se procederá a la integración de los servicios y funciones del IASAM y, además, que durante el período transitorio se adoptarán las medidas oportunas para la "plena coordinación funcional con el SAS" (Ley 8/1986). Por consiguiente, todo estaba preestablecido para que en 
la disposición adicional décima de la Ley de Presupuestos Generales de la Comunidad Autónoma para 1991 (Ley 6/1990), se estableciera, con un año y medio de retraso sobre lo previsto, la derogación de la ley de creación del IASAM, que finalmente deja de existir el 31 de Diciembre de 1990.

Posiblemente la normativa que hace desaparecer el IASAM debería haber merecido otro tipo de disposición legal que contemplara, por ejemplo, medidas más precisas para la correcta articulación de la integración de los servicios de salud mental en el Servicio de Salud Andaluz. Puede pensarse que la envergadura del SAS superaba con mucho la del IASAM, pero también se debería haber tenido en cuenta la enorme complejidad que supone la gestión de la salud mental de la población, en un sistema sanitario público, universal y equitativo, y por tanto no excluyente. Esto equivale a decir que no eran válidas soluciones "manicomiales" para pacientes gravemente afectados, pero también el tener en cuenta la importancia epidemiológica en la población general de otros trastornos, como los trastornos de ansiedad/depresión, muchas veces considerados "menores", pero causantes de grandes limitaciones y necesitados, en ocasiones de tratamientos de larga duración. Todo esto y muchas cosas más, como el sufrimiento en los allegados y en el entorno, la estigmatización - no solo de pacientes, también de familiares y profesionales-, la nula existencia de soportes socio-comunitarios, etc. no aconsejaba la medida adoptada en aquella ley de presupuestos (Dueñas, 2000).

Llegados a este punto, podríamos ahora hacer la siguiente reflexión de carácter excluyente: o se debería haber legislado de otra forma,- dando más tiempo al IASAM o dotándolo de vida indefinida-, o la integración de los órganos de gestión en el SAS debería de haber contemplado un mayor rango en la estructura organizativa, dada la envergadura de los problemas que acaba de señalarse.

Pero al margen de la normativa el IASAM estaba también abocado a integrarse en esa única red que es el Servicio Andaluz de Salud. Era un objetivo nuclear de la reforma el integrarnos en el sistema sanitario general, como señal de haber superado nuestra marginalidad. La opción por la integración quizá pecó de una visión simple e ingenua para afrontar estos asuntos, a pesar de las recomendaciones y las experiencias ya acaecidas en otras regiones del mundo (Leff, Thornicroft, Coxhead, \& Crawford, 1994; Murray y López, 1994) y del aumento de la demanda que se estaba detectando por organismos internacionales (World Health Organization, 1988). La red de salud mental era contemplada por el aparato sanitario general como algo tradicionalmente ajeno al mismo y, en consecuencia, con una mezcla de desconfianza y de agravios. Desconfianza de los modos de conducirse los pacientes, también de la capacidad de los profesionales; y los agravios sobrevenidos por haber sido un subsistema de salud con una profunda y rápida transformación en los últimos cinco años y un gran peso político en ese período.

No creemos equivocarnos mucho en este análisis. Baste recordar, por citar solo un ejemplo, lo que supuso, en relación con esta visión, el traslado de las unidades de corta estancia de los Hospitales psiquiátricos a los hospitales generales: los miedos, dificultades, espacios poco adaptados, etc, sin olvidar el impacto en los sistemas evaluativos (estancia media, índice de ocupación, etc.) que no podían ser entendidos por un sistema médico-quirúrgico que rentabilizaba al máximo estos indicadores, como expresión del progreso técnico y de gestión que suponían. En resumen, con este panorama era difícil aspirar a un alto rango dentro de la organización en la que, por otra parte, era ineludible integrarse.

\section{Referencias}

Corcés Pando, V., \& del Río, P. A. (1980). Aproximaciones a un año de transformación asistencial en el Hospital Psiquiátrico de Málaga. In M. González de Chávez (Org.), La transformación de la Asistencia Psiquiátrica (pp. 681-689). Madrid: Asociación Española de Neuropsiquiatría.

Dueñas, C. (2000). La Reforma Inacabada. Andalucía ¿Identidades y Diferencias en las Reformas Autonómicas? I Congreso Virtual de Psiquiatría . Recuperado de http://www.psiquiatria.com/congreso_old/mesas/mesa48/ mesa48.htm

González Álvarez, O., \& Fernández Sanabria, J. A. (1980). La experiencia del Hospital psiquiátrico de Huelva a través de seis documentos. In M. González de Chávez (Org.), La transformación de la Asistencia Psiquiátrica (pp. 517531). Madrid: Asociación Española de Neuropsiquiatría.

Instituto Andaluz de Salud Mental (1985). Situación jurídica de los pacientes psiquiátricos andaluces. Informe Técnico $n^{\circ} 1$. Sevilla: Consejería de Salud y Consumo.

Instituto Andaluz de Salud Mental (1988a). La Reforma Psiquiátrica en Andalucía 1984/1990. Serie Monográfica de Salud Mental (pp. 43-44). Sevilla: Consejería de Salud y Servicios Sociales.

Instituto Andaluz de Salud Mental (1988b). Bases generales para la atención comunitaria en Salud Mental. Consejería de Salud.

Instituto de Estadística y Cartografia de Andalucía (1986). Padrón Municipal de Habitantes. Recuperado de http://www.juntadeandalucia.es/ institutodeestadisticaycartografia/bd/indea/indea_VerTabla.jsp?tipo=T\&a $\mathrm{mb}=\mathrm{P} \&$ per $=\mathrm{A} \& \mathrm{~s}=49 \& \mathrm{i} 49=\mathrm{P}$

Leff, J.; Thornicroft, G.; Coxhead, N.; Crawford, C. (1994). The TAPS project: A five- year follow-up of long-stay psychiatric patients discharged to the community. British Journal of Psychiatry, 165(sup. 25), 13-17.

Ley n. 9 de 3 de julio de 1984 (1984, 3 de julio). Por la que se crea el Instituto Andaluz de la Salud Mental. Boletín Oficial de la Junta de Andalucía, $\mathrm{n}^{\circ}$ 67 del 13 de julio de 1984.

Ley n. 8 de 6 de mayo de 1986 (1986, 6 de mayo). Por la que se crea el Servicio Andaluz de Salud. Boletín Oficial de la Junta de Andalucía, $\mathrm{n}^{\circ} 41$ del 10 de Mayo de 1986.

Ley 6 de 29 de diciembre de 1990 (1990, 29 de didiembre). Presupuestos Generales de la Comunidad Autónoma para 1991. Boletín Oficial de la Junta de Andalucía, $\mathrm{n}^{\circ} 107$ del 31 de Diciembre de 1990.

López, M; Benítez, F; García, M. J., \& Weissman, L. C. (1998). Un programa para la supresión de los hospitales psiquiátricos públicos en Andalucía (1985-1995). In J. García, A. Espino, \& L. Lara (Orgs.), La Psiquiatría en la España de fin de siglo (pp. 101-116). Madrid: Díaz de Santos.

Murray, C. J. L, \& López, A. D. (1994). Global comparative assessment in the health sector. Disease burden, expenditures and intervention packages. Geneva:World Health Organization.

Pérez, F. (Org.). (2006). Dos décadas tras la Reforma Psiquiátrica. Madrid: Asociación Española de Neuropsiquiatría.

Piñero Ramirez, J. L., Mendez Marín, A., \& Murillo Esteve, J. R. (1980). El movimiento de los auxiliares en el Hospital psiquiátrico de Sevilla. In M. González de Chávez (Org.), La transformación de la Asistencia Psiquiátrica (pp. 619-639). Madrid: Asociación Española de Neuropsiquiatría.

World Health Organization [WHO] (1988). Prevention of mental, psychosocial and neurological disorders in the European Region. Geneva: World Health Organization Regional Office for Europe. 
José Carmona Calvo é psiquiatra, coordinador da Unidad de Salud Mental de Jerez, Unidad de Gestión Clínica de Salud Mental del Hospital General de Jerez. Servicio Andaluz de Salud. Endereço para correspondencia: C./ José Luis Díez n ${ }^{\circ} 14,5^{\text {a }}$ planta. Jerez de la Frontera, 11403. Cádiz, España. Telefones ++34 956-033627/ 628; Fax: ++34 956-033629.

E-mail: jose.carmona.calvo.sspa@juntadeandalucia.es

Francisco del Río Noriega é psiquiatra, director de la Unidad de Gestión Clínica de Salud Mental del Hospital General de Jerez. Servicio Andaluz de Salud. E-mail: francisco.rio.sspa@juntadeandalucia.es 